\title{
El trazado del icosakaidígono en el Clypeus Jupiter-Ammon de Tarragona (s. I) y la faz de Cristo del rosetón de la catedral de Orvieto (s. XIV)
}

\author{
The layout of icosakaidígono in the Jupiter-Ammon Clypeus of Tarragona \\ (It century) and the face of Christ of the Rosette in the Cathedral of Orvieto \\ (14 $4^{\text {th }}$ century)
}

J. Lluis i Ginovart $^{(*)}$, A. Samper ${ }^{(*)}$, S. Coll-Pla ${ }^{(*)}$, A. Costa-Jover ${ }^{(*)}$

\section{RESUMEN}

Las técnicas de captura masiva de datos, como el escáner láser terrestre y la fotogrametría, permiten analizar con precisión objetos próximos y otros no accesibles, mediante métodos estadísticos. Su aplicación ha permitido analizar desde el punto de vista geométrico las esculturas de la figura Clypeus de Tarragona, Jupiter-Ammon (s. I) y la del rosetón de la catedral de Orvieto con la faz de Cristo (s. XIv). El Clypeus de Tarragona y el rosetón de la catedral de Orvieto está divido en 22 partes, formando un icosakaidígono, polígono que no aparece en los Elementa de Euclides, ni en el Almagesto de Tolomeo, ni en las escasas Practica Geometriae de la época. Estos polígonos pueden ser construidos mediante la división angular heredada de los constructores de astrolabios y cuadrantes; así como también mediante una escuadra de dos brazos, con el apoyo reiterado de los catetos sobre la hipotenusa, creando así una rotación del instrumento u otros métodos directos. La conclusión del análisis de estas figuras es que, estadísticamente, ambas esculturas tienen una construcción geométrica igual al del Reliquiario del Corporale del Miracolo di Bolsena (1337-1338) de la catedral de Orvieto.

Palabras clave: Tarraco; catedral de Orvieto; Clypeus; geometría medieval; polígonos regulares; icosakaidígono.

\section{ABSTRACT}

The techniques of massive data capture, as the Terrestrial Laser Scanner and the digital photogrammetry, allow to accurately analyze close objects and others that are not accessible, by statistical methods. Its application has allowed assessing, from the geometric point of view, the sculptures of the figure Clypeus of Tarragona, Jupiter-Ammon ( $1^{\text {st }}$ century) and the one of the Orvieto's Cathedral rosette, with the face of Christ (14 century). The Clypeus of Tarragona and Orvieto's rosette are divided in 22 parts, forming a polygonal shape known as icosakaidígono. This polygon does not appear in the Elementa of Euclides, neither in the Almagesto of Ptolomeo, nor in the rare Practica Geometriae of the time. These polygons can be built by means of angular division inherited from astrolabe and quadrant builders. This polygon can be defined also through the rotation of the square, since the repeated support of the catheti on the hypotenuse allows the rotation of the tool, or other indirect methods. The conclusion of the analysis of these figures is that, statistically, both sculptures have a geometric construction equal to the Reliquiario del Corporale del Miracolo di Bolsena (1337-1338) of Orvieto's Cathedral.

Keywords: Tarraco; Orvieto Cathedral; clypeus; Medieval Geometry; regular polygon; icosakaidigon.

(*) Universidad Rovira y Virgili (Tarragona, España).

Persona de contacto/Corresponding author: recercapatrimoni@urv.cat (J. Lluis i Ginovart) ORCID: http://orcid.org/oooo-0001-5957-762X (J. Lluis i Ginovart); http://orcid.org/oooo-00o2-6194-3243 (A. CostaJover); http://orcid.org/oooo-0oo2-4718-5810 (S. Coll-Pla); http://orcid.org/oooo-0oo2-4795-2127 (A. Samper)

Cómo citar este artículo/Citation: Lluis i Ginovart, J., Samper, A., Coll-Pla, S., Costa-Jover, A. (2016). El trazado del icosakaidígono en el Clypeus Jupiter-Ammon de Tarragona (s. I) y la faz de Cristo del rosetón de la catedral de Orvieto (s. xiv). Informes de la Construcción, 69(545): e173, doi: http://dx.doi.org/10.3989/ic.16.048.

Copyright: (C) 2017 CSIC. Licencia / License: Salvo indicación contraria, todos los contenidos de la edición electrónica de Informes de la Construcción se distribuyen bajo una licencia de uso y distribución Creative Commons Attribution License (CC BY) Spain 3.o. 


\section{INTRODUCCIÓN Y OBJETIVO}

El ámbito de la investigación pretende analizar desde el conocimiento epistemológico el trazado geométrico de polígonos regulares en el mudo clásico y medieval, el cual no aparece en los dos textos de referencia de geometría culta, como son los Elementa de Euclides y el Almagesto de Tolomeo y tampoco en aquellos de aplicación práctica del Corpus agrimensorum del mundo romano o de las Practica Geometriae en el período gótico. El objetivo de la investigación es determinar el procedimiento de trazado geométrico de dos elementos escultóricos como el Clypeus de Tarragona (s. I) (Figura 1) y la de la faz de Cristo (s. xIv) (Figura 2) del rosetón de la catedral de Orvieto, ambos generados con la figura del icosakaidígono, o polígono de 22 lados.

Ambas figuras tienen la peculiaridad de estar dividas en 22 partes, formando un icosakaidígono, siendo una de las escasas figuras utilizadas en la tradición romana, como el mausoleo de Le Carceri Vecchie (s. I) de Capua. También es poco utilizada en el gótico, a excepción del rosetón de la catedral de Troia (s. XII), o la rotonda de Neuvy-Saint-Sépluche de Indre (s. XII).
El Clypeus de Tarragona es una pieza en parte reconstruida, depositada en el Museo Nacional Arqueológico de Tarragona (MNAT-117). La pieza fue esculpida en el siglo primero en mármol de Luni-Carrara y formaba parte de la decoración del ático del recinto de culto de Tarraco. El medallón tiene en su centro la representación de las deidades de Jupiter-Ammon (1), (2).

La construcción del rosetón de la catedral de Orvieto se realiza tras la modificación del proyecto original de la fachada principal de Lorenzo Maitini (c. 1275-1330), efectuada el año 1310 (3) por el escultor Andrea di Cione (c. 1308-1368), conocido como Andrea di Orcagna, modificando el diseño del rosetón de base octogonal (4), (5), (6). En el centro aparece representada la faz de Cristo sobre una crux immissa quadrata, rodeada de una corona con veintidós columnas. Tiene la misma estructura que un clypeus, pero a diferencia de éstos, que disponen de una ornamentación en forma vegetal, su corona se construye con 21 arcos trebolados.

Existen antecedentes de trazas geométricas de rosetones como los de Villard de Honnecourt (ca. 1220-1250) (BnF. ms. fr. 190093), quien dibuja el de la catedral de Nôtre-Dame de
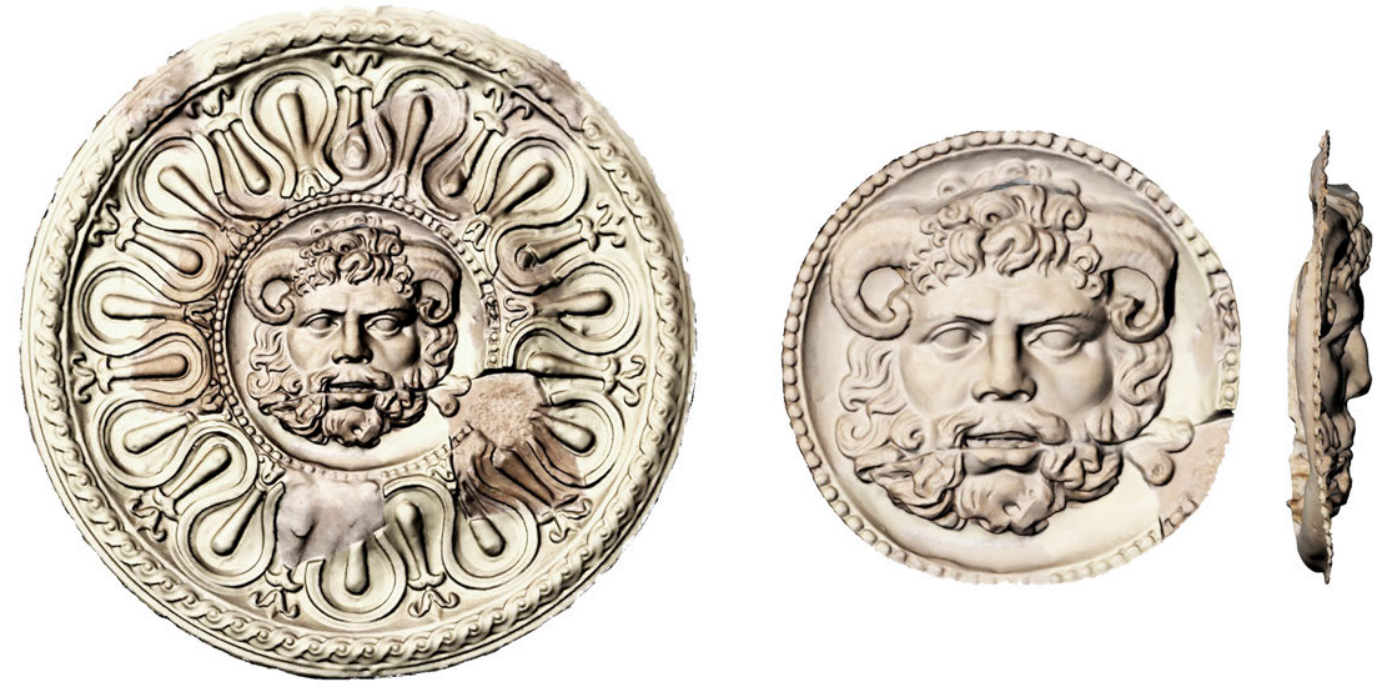

Figura 1. Clypeus de Tarragona, Jupiter-Ammon (s. I).
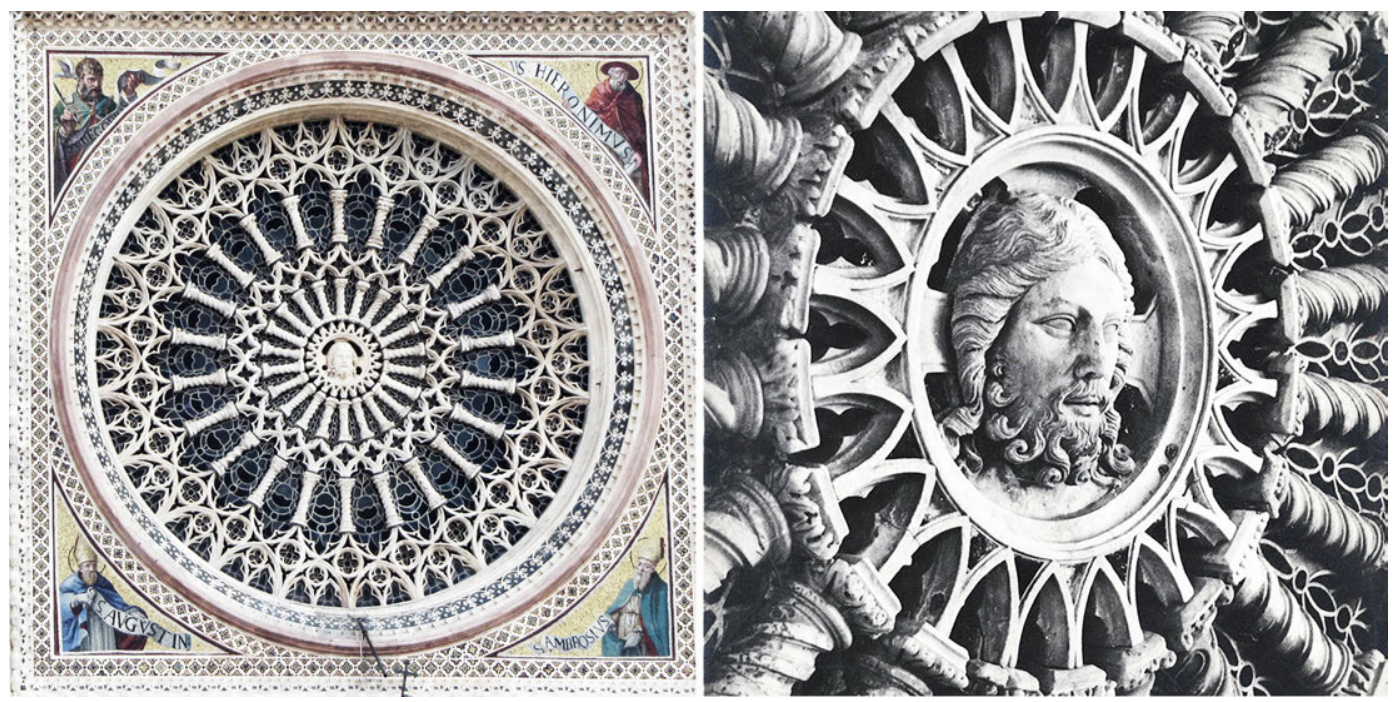

Figura 2. Rosetón Orvieto con la faz de Cristo (s. XIV). 
Chartres (fol. 15 v.), dividido en 12 partes, como también el de Lausanne (fol. 16 v.) (7), el del baptisterio de San Juan de Siena (S4) (8), y el del Plan B de la fachada de Estrasburgo (1255-1260) (9). En la misma catedral, en el Proyecto de 1365, la circunferencia está dividida en 36 partes (10). En el catálogo del Maestro WG (1560-1572), de Frankfurt, los diseños (WG 8. WG 22) están divididos en 8 partes, el (WG 15) en diez y el (WG 87) en 24 lados (11).

La división de la circunferencia en 22 partes es uno de los problemas que ha de abordar tanto el escultor romano como Andrea di Orcagna. Las construcciones geométricas que tienen como base las figuras del cuadrado, el pentágono, el hexágono y el octógono, se identifican en los Elementa de Euclides (326-265 a.C.) (12) y en el Almagesto de Ptolomeo (c. 85-165) (13), sin embargo, el polígono de 22 lados no aparece en estas referencias cultas.

\section{METODOLOGÍA}

A partir de una toma de datos, como elemento instrumental determinado en función de la accesibilidad de las esculturas, se propone un tratamiento estadístico de estos datos objetivos. Así los resultados obtenidos se comparan en función del error relativo de los 22 puntos que determinan la figura del icosakaidígono. Estos resultados se cotejan geométricamente con aquellos métodos operativos que se conocían en el período tardoclásico hasta el mundo gótico.

Existen antecedentes para el trazado del polígono de 22 lados heredados del mundo clásico, teniendo como base las aproximaciones del número $\mathrm{Pi}(\pi)$, conocidas en el mundo medieval a través de Arquímedes (c. 287 a.C.-c. 212 a.C.) (14). Los métodos están basados en la división del diámetro en $n$ partes, para luego abatirlos sobre la cuerda de la circunferencia. Sir Thomas Little Heath (1861-1940) determina en Grecia la relación (14/25) que fijaremos como $\mathrm{M}_{1,} \mathrm{y}$ la construcción del endecágono con la relación (2/7), método $\mathrm{M}_{2}$ (15). La Métrica (LI. XXIV), de Herón de Alejandría (c. 20-62), utiliza la relación de (7/25) (16). En el Clypeus de Tarragona (s. I) de Jupiter-Ammon se especula con la aproximación de (14/25) (17), ambos ejemplos según $\mathrm{M}_{1}$. El mausoleo de Le Carceri Vecchie (s. I) de Capua, de base circular con 22 módulos, tiene una relación (1/7), método $\mathrm{M}_{3}$ (18).

En el contexto medieval, en el álbum de Villard de Honnecourt (ca. 1220-1250) (BnF. ms. fr. 190093, fol. 20 v.), se construye una torre pentagonal, utilizando el movimiento de una escuadra (19). También lo es la referencia de la tumba de Saint-Ouen (ca. 1300), donde el ábaco en la mano del arquitecto ha sido asimilado a la construcción de polígonos regulares inscritos en una circunferencia (20). Otra alusión es la de Diego López Arenas (+c. 1640) en la Primera y segunda parte de la carpintería hecho por Diego López de Arenas (1619), donde define el cartabón para la traza de polígonos regulares (21). Se requiere así el método de las escuadras utilizando las proporciones entre los brazos del (16/25), método $\mathrm{M}_{4}$ y la de (1/7) como $\mathrm{M}_{5}$. Estos métodos son reiterativos y permiten ajustes.

Otra tesis para la construcción de estos polígonos sería la división angular de las Practica Geometriae, utilizados para la construcción del trazado de astrolabios o cuadrantes astronómicos, método $\mathrm{M}_{6}$. Caso de Hernán de Contracto (1013-1050) en la De mensura astrolabii (c. 1050) (22), la Practica Geo- metriae (1125-1130) de Hugo de San Víctor (ca. 1100-1141) (23), el Artis cuiuslibet consummatio (c. 1193-c. 1220) atribuido a Gérard de Bruxelles (f. 1220) (24), el Quadrans Vetus de Robert Anglès (c. 1240-c. 1271) (25) o la Practica Geometriae (1346) de Dominicus de Clavasio (+c. 1362) (26).

Otra posibilidad es el conocimiento de las geometrías cultas esencialmente concentradas en los Elementa y el Almagesto donde, aunque no aparezca el undecágono, sí se podría construir mediante la intercalación de dicha figura entre los polígonos de 20 y de 24 lados, trazados a partir del pentágono y hexágono. También como consecuencia de éstos, como las figuras pes Arenis, cauda Pavonis, Medriatix, Pax et Concordia o la Demonis y trazadas con compás (27). Figuras próximas a la hipótesis geométrica del Reliquiario del Corporale del Miracolo di Bolsena (1337-1338) de Orvieto, de Ugnolino di Vieri, método $M_{7}(28)$.

La toma y captura de los datos geométricos se realiza por métodos diferentes en función de la accesibilidad de las esculturas. En el caso de la faz de Cristo (s. XIv) del rosetón de la catedral de Orvieto éstos son captados mediante técnicas de escáner láser, puesto que el objeto es inaccesible (29), (30). La fiabilidad de estos datos ha sido ampliamente testeada (31), (32) permitiendo el estudio detallado de objetos lejanos.

Otras técnicas de aproximación a objetos cercanos, de pequeña y mediana escala, permiten el análisis preciso de su geometría a partir de la fotografía. Será el caso de las técnicas fotogramétricas (33) como la de Close Range Photogrammetry (CRP) (34) y, sobre todo, la de Structure From Motion (SFM), desarrollada más recientemente, y que permite una operativa sencilla para reproducir la geometría y textura de objetos de diversas escalas (35), (36). La digitalización de la figura Clypeus de Tarragona (s. I) se ha realizado mediante fotogrametría con el programa 123Catch de Autodesk. Se trata de un software gratuito basado en el sistema SFM. La precisión de este software presenta algunas limitaciones respecto a otros programas comerciales, especialmente en el caso de levantamientos de escala arquitectónica (37), (38), donde la definición de la escala métrica afecta a la precisión final del modelo. El objeto analizado en este caso tiene una medida relativamente pequeña, y el fotografiado pudo realizarse a una distancia adecuada, por lo que las distorsiones métricas son muy reducidas. Además, el tamaño del Clypeus también provoca que las desviaciones causadas por el escalado del objeto tengan una incidencia mínima en los resultados del estudio. Este programa ofrece una gran facilidad de uso, y no es necesario disponer de una gran capacidad de computación para obtener resultados, como sucede con otros programas comerciales (39).

\section{RESULTADOS}

El levantamiento topográfico del rosetón de Orvieto se ha realizado mediante un escáner láser terrestre, modelo Leica ScanStation P20, con 11 posicionamientos, 8 estaciones a nivel peatonal y 3 en la cubierta del Museo Claudio Faina, situado frente a la fachada principal de la catedral. Las nubes de puntos se han procesado con los programas «Cyclone» $\mathrm{y}$ «3DReshaper», con el fin de obtener una imagen de gran precisión y a escala métrica del rosetón.

La digitalización del Clypeus se ha realizado mediante fotogrametría con el programa 123Catch de Autodesk, que per- 
mite la obtención de un modelo tridimensional de forma automatizada, a partir del fotografiado exhaustivo de la pieza desde distintas posiciones. El solape entre las fotografías es total en la toma de datos, es decir, cada fotografía captura completamente el Clypeus, desde distintas posiciones. Se ha utilizado para ello una cámara Nikkon D700o, con un objetivo Tamron (AF 17-50mm, F/2.8). Autodesk no proporciona datos sobre los algoritmos de alineación de las fotografías ni de calibración de la cámara. Una vez obtenida la malla triangular, ésta se refina con el programa Rhinoceros, con el que también se genera la ortoimagen a escala métrica. El escalado de la pieza se realiza a partir de medidas tomadas manualmente in situ.

Una vez obtenidos ambos modelos y generadas las ortoimágenes, se coordenan todos los puntos con el software vectorial AutoCAD (Figura 3). Los 22 puntos para el estudio se han identificado de forma organoléptica. En el caso del rosetón la elección entre todos los puntos topográficos se ha afinado detectando los 22 puntos más próximos al extremo de las esculturas, teniendo una dispersión menor de 0,002 m. El rango de precisión de la toma de datos, elección de puntos y su tratamiento lo situamos en (0,003-0,004 m), totalmente aceptable para un tratamiento matemático de esta operativa. En el caso del Clypeus la elección de los puntos se ha realizado sobre la escultura, con una dispersión también inferior a 0,002 m. La precisión del levantamiento y la definición de las coordenadas para el estudio se establecen en el mismo rango que el del rosetón (0,003-0,004 m). Se asume que las incidencias sobre los elementos, como los errores de ejecución o la reconstrucción del Clypeus, pueden alterar el método inicial de trazado, por ello se acude a una metodología que es una simbiosis entre los métodos geométricos y estadísticos. Por ello, el primer paso para este análisis geométrico y estadístico es calcular la circunferencia de regresión de los 22 puntos que configuran ambos elementos.

Se asigna a los 22 puntos para cada caso $\mathrm{v}_{\mathrm{i}}(\mathrm{i}=1 \div 22)$, de las 22 arcadas perimetrales del medallón del rosetón y de la anilla del Clypeus (Figura 4). En ambos elementos se observan que de los 22 vértices $v_{i}$, vemos que no existe ninguna circunferencia que pase por más de 3 puntos. Aplicando ecuaciones normales de Gauss $\left(\mathrm{x}_{\mathrm{i}}, \mathrm{y}_{\mathrm{i}}\right)$ de estos $22 \mathrm{v}_{\mathrm{i}}$ (Tabla 1) y encontramos las ecuaciones de las circunferencias $[1,2]$ de regresión cuadrática $C$ para el caso del rosetón [1] y para el Clypeus [2].

$$
0,005958\left(x^{2}+y^{2}\right)-0,114367 x-0,103766 y+1=0
$$

Encontramos que la posición del centro del rosetón de $C_{1}$ es $(9,5982,8,7084)$ y su radio $\mathrm{R}=0,3378 \mathrm{~m}$.

$$
0,003601\left(x^{2}+y^{2}\right)-0,108388 x-0,051596 y+1=0
$$

Encontramos que la posición del centro del Clypeus de $C_{2}$ es $(15,049407,7,164007)$ y su radio $\mathrm{R}=0,3349 \mathrm{~m}$.

En esta regresión cuadrática por circunferencia $C_{1}$ se rechaza la hipótesis nula $\mathrm{h}_{\mathrm{o}}$ de no correlación cuadrática por circunferencia aplicando el test F de Ficher-Snedecor con nivel de significancia $\alpha=1-\mathrm{G}(2186,809 ; 3,18)=0$; y el tanto por ciento de coordenadas y explicadas por las coordenadas $\mathrm{x}$ de la regresión cuadrática por circunferencia $C_{1}$ es el coeficiente de determinación de Pearson ajustado del $\eta_{\text {adj }}^{2} \times 100=99,999903 \%$ debido a que $\eta_{\text {adj }}^{2}=1-\left(1-\eta^{2}\right) \frac{22-1}{22-3-1}$, donde

$$
\eta^{2}=\frac{\sum_{i=1}^{22}\left(\hat{y}_{i}-\bar{y}\right)^{2}}{\sum_{i=1}^{22}\left(y_{i}-\bar{y}\right)^{2}}=0,99999917
$$

es el coeficiente de determinación de Pearson de $C_{1}$, donde $\bar{y}=\sum_{i=1}^{22} \frac{y_{i}}{22}, y \hat{y}_{i}$ es la coordenada y correspondiente a la $x_{i}$ obtenida por la regresión de la circunferencia $C_{1}$. La distribución $\mathrm{G}\left(\mathrm{x} ; \mathrm{d}_{1}, \mathrm{~d}_{2}\right)=\mathrm{I}_{\frac{\mathrm{d}_{1} \mathrm{x}}{\mathrm{d}_{1} \mathrm{x}+\mathrm{d}_{2}}}\left(\frac{\mathrm{d}_{1}}{2}, \frac{\mathrm{d}_{2}}{2}\right)$ es la distribución de Ficher-

Snedecor donde I es la función beta incompleta regularizada. Dicho de otra forma, $C_{1}$ ofrece una correlación cuadrática por circunferencia entre las coordenadas de los 22 puntos del rosetón con probabilidad total prácticamente 1 y esta correlación explica al 99,999903\% las coordenadas de los mismos. Por lo tanto, no sólo es la circunferencia que más aproxima sino que aproxima a la perfección y por ello podemos considerarla como circunferencia inicial del modelo real del rosetón.
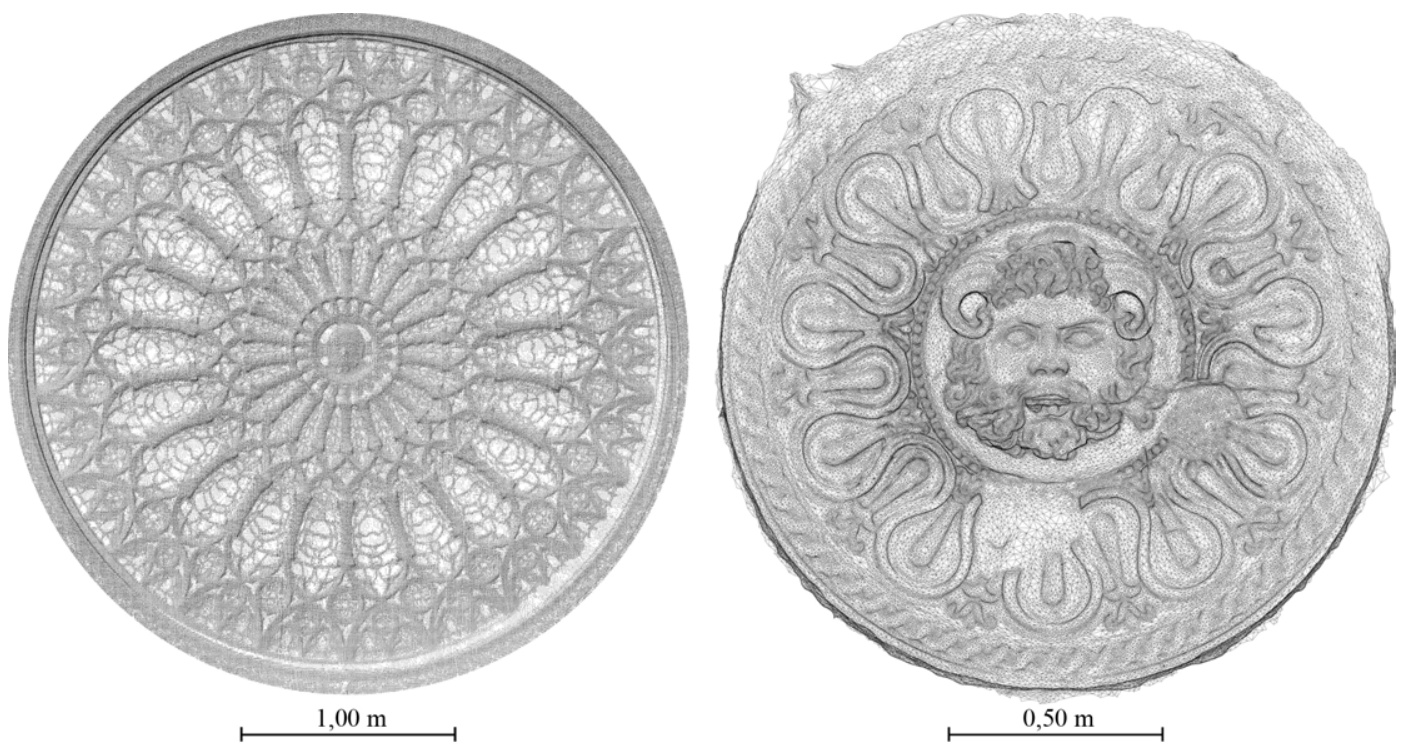

Figura 3. Levantamiento fachada principal de la catedral de Orvieto y Clypeus de Tarragona. 

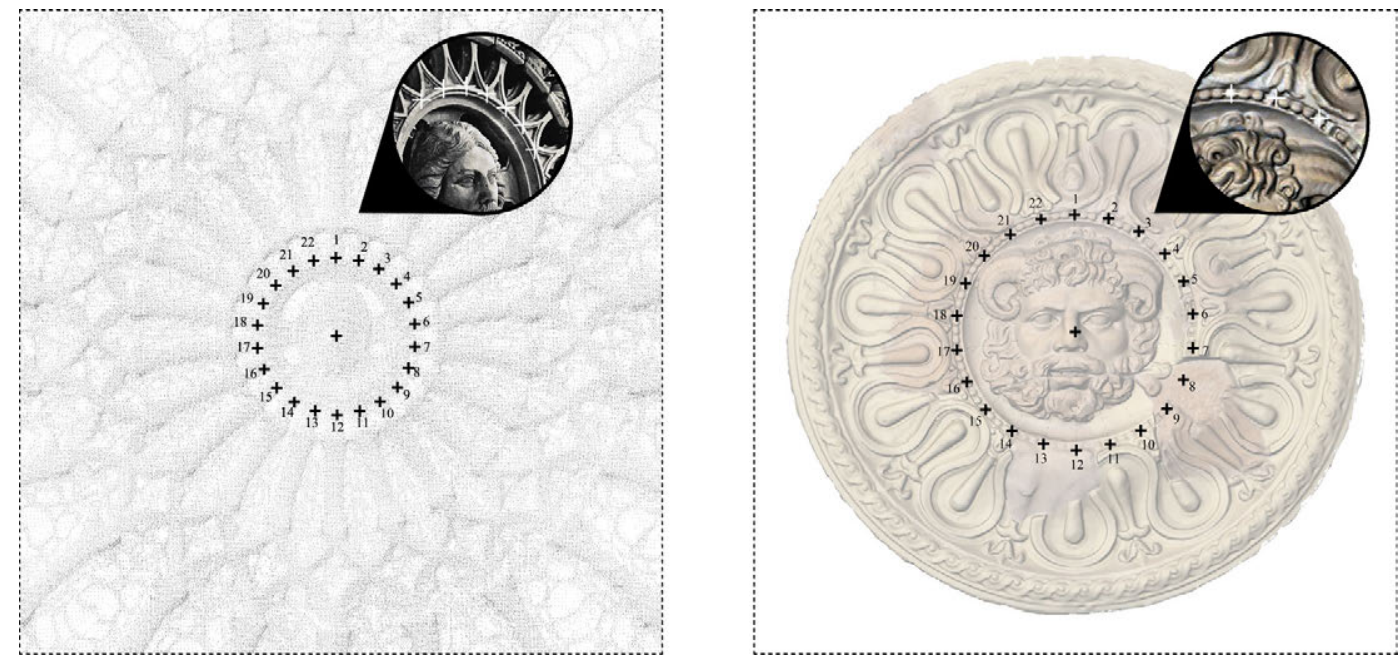

Figura 4. Selección de los 22 puntos para ambos elementos arquitectónicos considerados en el análisis.

Tabla 1. Coordenadas de los 22 vértices y parámetros relativos de la pieza central del rosetón de Orvieto y del Clypeus de Tarragona.

\begin{tabular}{|c|c|c|c|c|c|c|}
\hline \multirow[b]{2}{*}{$\mathbf{i}$} & \multicolumn{3}{|c|}{ Pieza central del rosetón de Orvieto } & \multicolumn{3}{|c|}{ Clypeus de Tarragona } \\
\hline & $\begin{array}{l}\text { Coordenadas } \\
\left(x_{i}, y_{i}\right) \text { de } v_{i}\end{array}$ & $\begin{array}{l}\text { Amplitud en } \\
\text { radians } \varphi_{\mathrm{i}}\end{array}$ & $\begin{array}{l}\text { Longitud } \\
{\text { lado } m_{i, i+1}}^{2}\end{array}$ & $\begin{array}{l}\text { Coordenadas } \\
\left(x_{i}, y_{i}\right) \text { de } v_{i}\end{array}$ & $\begin{array}{l}\text { Amplitud en } \\
\text { radians } \varphi_{\mathrm{i}}\end{array}$ & $\begin{array}{l}\text { Longitud } \\
{\text { lado } \mathrm{m}_{\mathrm{i}, \mathrm{i}+\mathbf{1}}}^{\text {L }}\end{array}$ \\
\hline 1 & $(9,5960,9,0424)$ & 0,29240 & o,0977 & $(15,0519,7,5058)$ & 0,26372 & 0,0895 \\
\hline 2 & $(9,6931,9,0313)$ & 0,27955 & 0,0941 & $(15,1401,7,4904)$ & 0,26358 & 0,0887 \\
\hline 3 & $(9,7797,8,9944)$ & 0,29022 & 0,0980 & $(15,2207,7,4533)$ & 0,28589 & 0,0959 \\
\hline 4 & $(9,8541,8,9306)$ & 0,28013 & 0,0949 & $(15,2957,7,3937)$ & 0,28120 & 0,0945 \\
\hline 5 & $(9,9073,8,8520)$ & 0,28451 & 0,0964 & $(15,3506,7,3167)$ & 0,30585 & 0,1026 \\
\hline 6 & $(9,9336,8,7593)$ & 0,28636 & 0,0968 & $(15,3810,7,2187)$ & 0,29031 & 0,0967 \\
\hline 7 & $(9,9339,8,6625)$ & 0,28093 & 0,0947 & $(15,3783,7,1220)$ & 0,28779 & 0,0967 \\
\hline 8 & $(9,9066,8,5718)$ & 0,28164 & 0,0953 & $(15,3608,7,0269)$ & 0,25042 & 0,0851 \\
\hline 9 & $(9,8595,8,4890)$ & 0,28556 & 0,0966 & $(15,3176,6,9536)$ & 0,28733 & 0,0968 \\
\hline 10 & $(9,7851,8,4274)$ & 0,28553 & 0,0958 & $(15,2428,6,8922)$ & 0,33421 & 0,1101 \\
\hline 11 & $(9,6979,8,3876)$ & 0,29327 & 0,0983 & $(15,1412,6,8497)$ & 0,27176 & 0,0880 \\
\hline 12 & $(9,6009,8,3714)$ & 0,28468 & 0,0951 & $(15,0534,6,8438)$ & 0,28156 & 0,0907 \\
\hline 13 & $(9,5074,8,3887)$ & 0,29306 & 0,0975 & $(14,9630,6,8507)$ & 0,30603 & 0,0996 \\
\hline 14 & $(9,4175,8,4263)$ & 0,28766 & 0,0965 & $(14,8709,6,8887)$ & 0,29231 & 0,0956 \\
\hline 15 & $(9,3425,8,4871)$ & 0,28101 & 0,0950 & $(14,7988,6,9515)$ & 0,29244 & 0,0955 \\
\hline 16 & $(9,2893,8,5658)$ & 0,28065 & 0,0954 & $(14,7623,6,9139)$ & 0,28960 & 0,0943 \\
\hline 17 & $(9,2605,8,6568)$ & 0,28794 & 0,0979 & $(14,7248,7,1245)$ & 0,31488 & 0,1036 \\
\hline 18 & $(9,2605,8,7547)$ & 0,27868 & 0,0948 & $(14,7231,7,2280)$ & 0,22980 & 0,0763 \\
\hline 19 & $(9,2853,8,8462)$ & 0,28084 & 0,0953 & $(14,7458,7,3008)$ & 0,36610 & 0,1225 \\
\hline 20 & $(9,3386,8,9252)$ & 0,28639 & 0,0960 & $(14,8106,7,4048)$ & 0,25309 & 0,0853 \\
\hline 21 & $(9,4130,8,9858)$ & 0,29301 & 0,0977 & $(14,8799,7,4545)$ & 0,24308 & 0,0823 \\
\hline 22 & $(9,5005,9,0292)$ & 0,28918 & 0,0965 & $(14,8654,7,4150)$ & 0,29223 & 0,0994 \\
\hline
\end{tabular}

De manera similar y para el caso de Clypleus de Tarragona, en la regresión cuadrática por circunferencia $C_{2}$ se rechaza la hipótesis nula $\mathrm{h}_{\mathrm{o}}$ de no correlación cuadrática por circunferencia aplicando el test $\mathrm{F}$ de Ficher-Snedecor con nivel de significancia $\alpha=1-\mathrm{G}(354,263 ; 3,18)=0$, y el tanto por ciento de coordenadas y explicadas por las coordenadas $\mathrm{x}$ de la regresión cuadrática por circunferencia $C_{2}$ es el coeficiente de determinación de Pearson ajustado del $\eta_{\text {adj }}^{2} \times 100=100 \%$ debido a que $\eta_{\text {adj }}^{2}=1-\left(1-\eta^{2}\right) \frac{22-1}{22-3-1}$. En resumen, $C_{2}$ ofrece una correlación cuadrática por circunferencia entre las coordenadas de los 22 puntos del Clypeus con probabilidad total 1 y esta correlación explica al $100 \%$ las coordenadas de los mismos.
Las coordenadas $\left(\mathrm{x}_{\mathrm{i}}, \mathrm{y}_{\mathrm{i}}\right)$ de los 22 vértices $\mathrm{v}_{\mathrm{i}} \mathrm{y}$ las amplitudes $\varphi_{\mathrm{i}}$ de los ángulos $\angle\left(\overrightarrow{\mathrm{Ov}_{\mathrm{i}}}, \overrightarrow{\mathrm{Ov}_{\mathrm{i}+1}}\right)-\mathrm{i}=1 \div 21 \mathrm{y} \varphi_{22}$ es amplitud del ángulo $\angle\left(\overrightarrow{\mathrm{Ov}_{22}}, \overrightarrow{\mathrm{Ov}_{1}}\right)$ - de la zona central del rosetón y del Clypeus son los mostrados en la Tabla 1. Sin embargo, a fin de poder comparar los métodos con el rosetón, escalaremos las circunferencias $C_{1}$ y $C_{2}$ para que su radio sea $\mathrm{R}=1 \mathrm{~m}$ al igual que el radio de todos los métodos de estudio y, además, trasladaremos ambos elementos para que sus centros de $C_{1} \mathrm{y}$ $\mathrm{C}_{2}$ sean el origen de coordenadas $(0,0)$.

Analizando las diferencias entre la magnitud de para cada posible método de trazado $\left(\mathrm{M}_{1} \ldots \mathrm{M}_{7}\right)$ y el valor teórico del lado 
del icosakaidígono, se pretende obtener resultados de base estadística como un método complementario a la historiografía o al conocimiento epistemológico de la pervivencia de la cultura clásica en el rosetón gótico.

\subsection{Análisis y construcción de métodos}

Se van a analizar las hipótesis que pudieran ser conocidas en la construcción del icosakaidígono o el endecágono en el período del siglo i al siglo xiv. Están agrupadas en aquellos métodos derivados de las aproximaciones de $\mathrm{Pi}(\pi), \mathrm{M}_{1}, \mathrm{M}_{2} \mathrm{y} \mathrm{M}_{3}$. Los construidos mediante escuadras los $\mathrm{M}_{4}$ y $\mathrm{M}_{5}$ de los que se tienen constancia en el mundo medieval, el de la división angular $\mathrm{M}_{6}$ de los constructores de instrumentos astronómicos medievales. Finalmente el método directo $\mathrm{M}_{7}$, el cual posiblemente se utilizó en la misma época para la construcción del rosetón, así como también fue utilizado en el Reliquiario del Corporale del Miracolo di Bolsena.

\subsection{Método $M_{1}, M_{2} y_{3}$}

Las construcciones $M_{1}, M_{2}$ y $M_{3}$ se determinan a partir de una circunferencia de centro $\mathrm{O}^{3}$ y diámetro $\overline{\mathrm{AB}}$. Se divide el diámetro en $\mathrm{N}$ partes, con la unidad $\mathrm{u}$, con centro $\mathrm{A}$ y radio $\mathrm{n}$ veces $\mathrm{u}$, se traza un arco de circunferencia que corta la circunferencia en el punto C. Con el segmento $\overline{\mathrm{AC}}$ del polígono inscrito en la circunferencia se traza un arco de radio $\overline{\mathrm{AC}}$ y centro $\mathrm{C}$, encontrando así los vértices del polígono. Así se construyen de forma aproximada del endecágono con las relaciones 14/25 $\left(\mathrm{M}_{1}\right), 2 / 7\left(\mathrm{M}_{2}\right)$ y del icosakaidígono 1/7 $\left(\mathrm{M}_{3}\right)$ (Figura 5).

Con el método $\mathrm{M}_{1}$ de construcción del endecágono y realizando la bisectriz de cada ángulo se obtiene el icosakaidígono configurado con 20 ángulos $\alpha$ y 2 ángulos $\beta(2 \pi=20 \alpha+2 \beta)$, cuyas ecuaciones analíticas son las siguientes:

$$
\alpha=\operatorname{arcsen} \frac{7}{25} \text { y } \beta=\pi-10 \operatorname{arcsen} \frac{7}{25} .
$$

Además, las coordenadas de los 22 vértices son:

$$
u_{1,1}=(1,0) ; u_{n+1,1}=(\cos n \alpha, \text { sen } n \alpha)
$$

$\operatorname{con} \mathrm{n}=1 \div 20 \mathrm{y} \mathrm{u}_{22,1}=(\cos (20 \alpha+\beta)$, $\operatorname{sen}(20 \alpha+\beta))$, Tabla 2 .

Con el método $\mathrm{M}_{2}$ de construcción del endecágono y realizando la bisectriz de cada ángulo se obtiene el icosakaidígono configurado con 20 ángulos a y 2 ángulos $\beta(2 \pi=20 \alpha+2 \beta)$, cuyas ecuaciones analíticas son las siguientes:

$$
\alpha=\operatorname{arcsen} \frac{2}{7} \text { y } \beta=\pi-10 \arcsin \frac{2}{7} .
$$

Además, las coordenadas de los 22 vértices son:

$$
\mathrm{u}_{1,2}=(1,0) ; \mathrm{u}_{\mathrm{n}+1,2}=(\cos \mathrm{n} \alpha, \operatorname{sen} \mathrm{n} \alpha)
$$

$\operatorname{con} \mathrm{n}=1 \div 20 \mathrm{y} \mathrm{u}_{22,1}=(\cos (20 \alpha+\beta), \operatorname{sen}(20 \alpha+\beta))$.

El icosakaidígono realizado con el método $\mathrm{M}_{3}$ está configurado con 21 ángulos $\alpha$ y 1 ángulo $\beta(2 \pi=21 \alpha+\beta)$, cuyas ecuaciones analíticas son las siguientes:

$$
\alpha=2 \operatorname{arcsen} \frac{1}{7} \text { y } \beta=2 \pi-42 \operatorname{arcsen} \frac{1}{7} .
$$

Además, las coordenadas de los 22 vértices son:

$$
u_{1,3}=(1,0) y u_{n+1,3}=(\cos n \alpha, \text { sen } n \alpha) \operatorname{con} n=1 \div 21 \text {. }
$$

\subsection{Método $M_{4}, M_{5}$}

La construcción mediante rotación de escuadras $\left(\mathrm{M}_{4}\right)$ y $\left(\mathrm{M}_{5}\right)$ tendrá como base la hipótesis de la construcción de la torre del Villard de Honnecourt (fol. 20 r.c.), Par chu portait om one toor a chinc arestes, construida mediante una escuadra que va situándose por los puntos medios y sextos (1/3-0,77). Otro método que proponemos se realiza con una escuadra de proporciones de (2/6), con la que se situará una alineación entre el segundo punto de un cateto y el extremo del otro, colocándose sobre esta alineación el nuevo lado del pentágono.

Otra tesis sería la posibilidad de construir un polígono a partir de un ábaco de la tumba de Saint-Ouen. La hipótesis comprueba que el punto 1 determina la diagonal de un cuadrado, el punto 2 la diagonal de un rectángulo de (4/5), el punto 3 el lado de un triángulo equilátero y sobre el punto 4 la pendiente de una recta de un tercio. Para construir un polígono determinado debían de conocerse las proporciones de los catetos de las escuadras. La proporción 4/12 servía para la construcción del pentágono; 4/7 permitía construir el hexágono; 4/5 permitía construir aproximadamente el heptágono; 4/4 permitía construir el octógono; 8/11 permitía construir el decágono. De esta manera la proporción $16 / 25$ permitía la construcción del endecágono, método $\left(M_{4}\right)$ y la 1/7 la construcción de un polígono de 44 lados $\left(M_{5}\right)$ (Figura 6$)$.

Así, este método sugiere la aproximación de los principales polígonos regulares de acuerdo con el siguiente cuadro, donde 1 es el lado desigual, $\alpha^{\circ}$ el ángulo desigual y $\varepsilon \%$ el error relativo frente al polígono regular (Tabla 3).
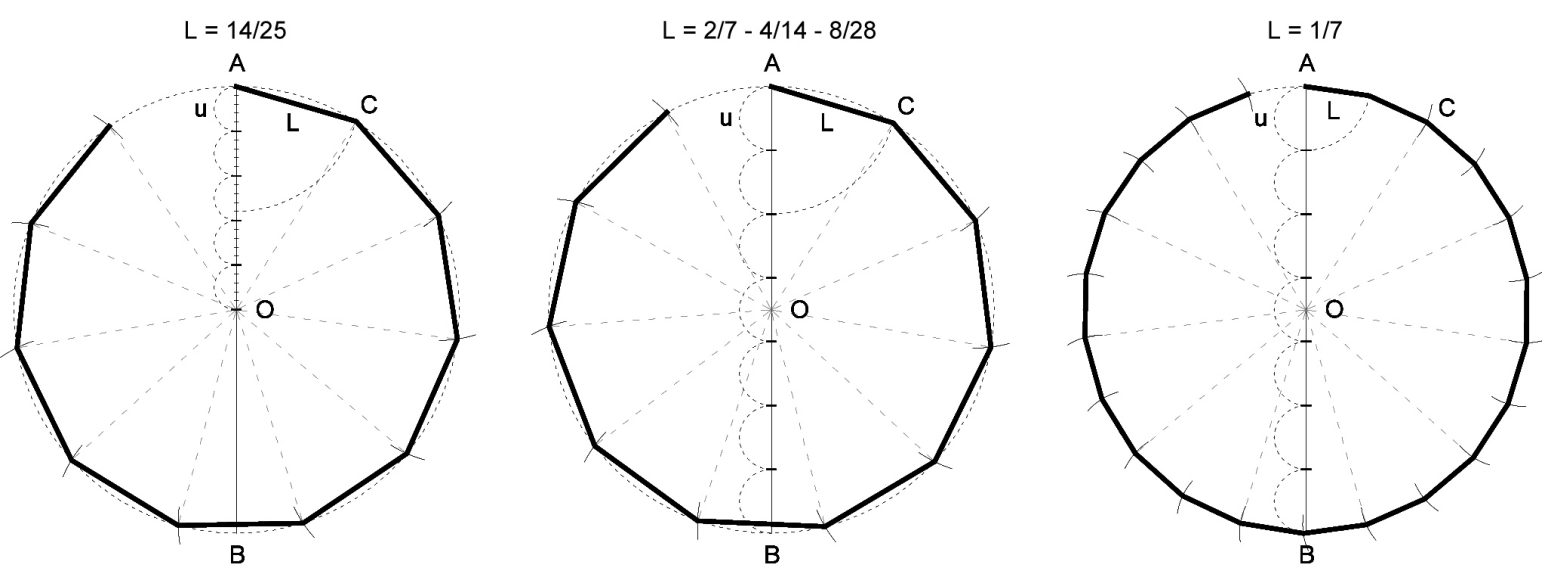

Figura 5. Resolución gráfica de los métodos $\mathrm{M}_{1}, \mathrm{M}_{2} \mathrm{y}_{3}$. 
Tabla 2. Correlaciones de comparación entre $\mathrm{M}_{4}$ y las coordenadas del rosetón y del Clypeus.

\begin{tabular}{|c|c|c|c|c|c|c|}
\cline { 2 - 7 } \multicolumn{1}{c|}{} & \multicolumn{2}{c|}{ Correlaciones de comparación del rosetón } & \multicolumn{2}{c|}{ Correlaciones de comparación del Clypeus } \\
\hline $\mathbf{i}$ & $\mathbf{P}$ & $\eta_{\text {adj }}^{2} \times \mathbf{1 0 0}$ & $\mathbf{d}$ & $\mathbf{P}$ & $\eta_{\text {adj }}^{2} \times \mathbf{1 0 0}$ & $\mathbf{d}$ \\
\hline 1 & 0,999807 & 99,959459 & 0,0038 & 0,994347 & 98,817581 & 0,0038 \\
\hline 2 & 0,999893 & 99,977586 & 0,0039 & 0,994825 & 98,917319 & 0,0035 \\
\hline 3 & 0,999812 & 99,960471 & 0,0039 & 0,994548 & 98,859573 & 0,0032 \\
\hline 4 & 0,999861 & 99,970950 & 0,0040 & 0,994533 & 98,856342 & 0,0030 \\
\hline 5 & 0,999780 & 99,953876 & 0,0041 & 0,994439 & 98,836701 & 0,0028 \\
\hline 6 & 0,999750 & 99,947561 & 0,0041 & 0,994752 & 98,902082 & 0,0027 \\
\hline 7 & 0,999762 & 99,950182 & 0,0041 & 0,994760 & 98,903797 & 0,0027 \\
\hline 8 & 0,999676 & 99,932032 & 0,0042 & 0,994732 & 98,897815 & 0,0028 \\
\hline 9 & 0,999583 & 99,912475 & 0,0041 & 0,994149 & 98,776269 & 0,0030 \\
\hline 10 & 0,999586 & 99,913281 & 0,0041 & 0,994195 & 98,785924 & 0,0032 \\
\hline 11 & 0,999599 & 99,915902 & 0,0041 & 0,994585 & 98,867293 & 0,0036 \\
\hline 12 & 0,999776 & 99,953026 & 0,0040 & 0,994706 & 98,892481 & 0,0039 \\
\hline 13 & 0,999766 & 99,950963 & 0,0040 & 0,994663 & 98,883575 & 0,0042 \\
\hline 14 & 0,999873 & 99,973458 & 0,0039 & 0,994443 & 98,837677 & 0,0046 \\
\hline 15 & 0,999888 & 99,976443 & 0,0038 & 0,994245 & 98,796408 & 0,0048 \\
\hline 16 & 0,999820 & 99,962323 & 0,0038 & 0,957779 & 91,330812 & 0,0046 \\
\hline 17 & 0,999732 & 99,943857 & 0,0037 & 0,994050 & 98,755681 & 0,0050 \\
\hline 18 & 0,999767 & 99,951115 & 0,0037 & 0,992762 & 98,487294 & 0,0050 \\
\hline 19 & 0,999628 & 99,921983 & 0,0037 & 0,994768 & 98,905485 & 0,0049 \\
\hline 20 & 0,999513 & 99,897804 & 0,0037 & 0,990603 & 98,038199 & 0,0047 \\
\hline 21 & 0,999546 & 99,904717 & 0,0037 & 0,992894 & 98,514734 & 0,0044 \\
\hline 22 & 0,999736 & 99,944539 & 0,0037 & 0,940256 & 87,842605 & 0,0043 \\
\hline Media & 0,999760 & 99,949672 & 0,0039 & 0,990047 & 97,941166 & 0,0039 \\
\hline
\end{tabular}

$4 / 12$
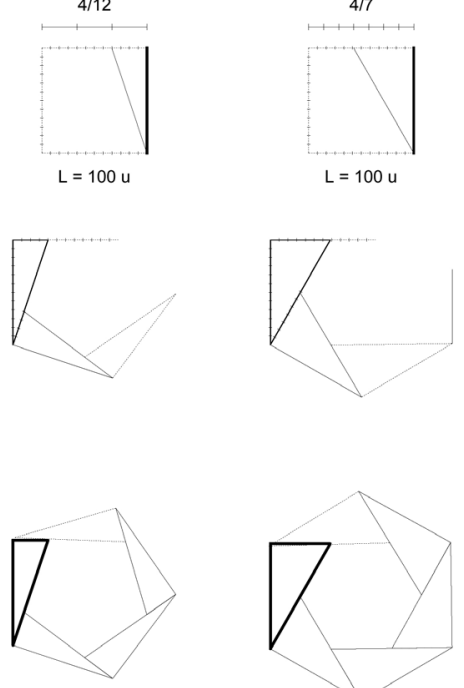

$L^{\prime}=102.6149 u$
6

$4 / 7$

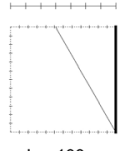

$L=100 u$
7

$4 / 5$

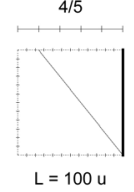

10

$8 / 11$

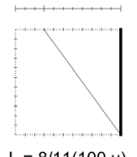

$L=8 / 11(100 u)$
11

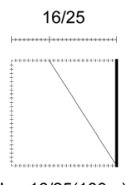

$L=16 / 25(100 u)$
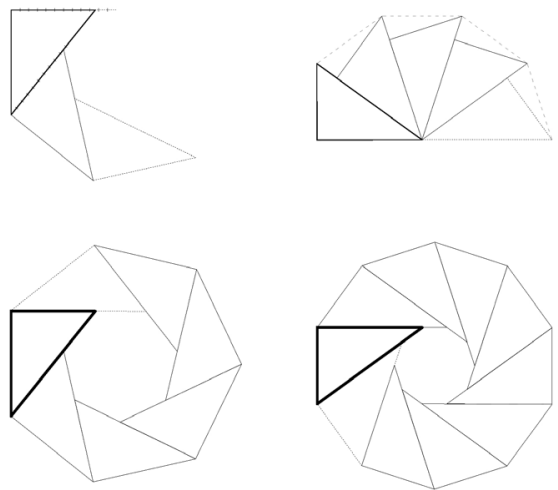

$L^{\prime}=101.1218 u$

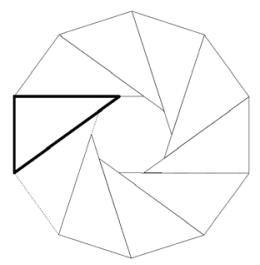

$L^{\prime}=72.1928 u$
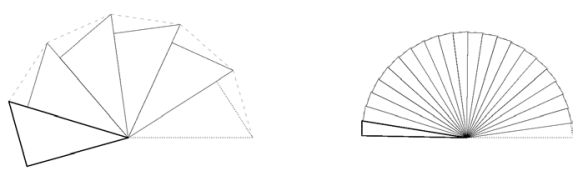

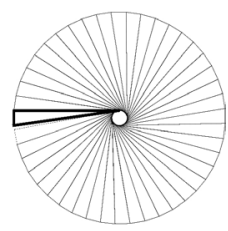

$L^{\prime}=18.2743 u$

Figura 6. Metodología de construcción de polígonos regulares mediante rotación de escuadras.

Tabla 3. Construcción de polígonos regulares mediante rotación de escuadras.

\begin{tabular}{|c|c|c|c|c|c|c|}
\hline N.o lados & Proporción & Longitud L & Longitud L' & $\boldsymbol{\varepsilon \%}$ & $\boldsymbol{\alpha}$ & $\boldsymbol{\alpha}^{\prime}$ \\
\hline 5 & $4 / 12$ & $100 \mathrm{u}$ & $102,614 \mathrm{u}$ & 2,614 & $71,565^{\circ}$ & $73,739^{\circ}$ \\
\hline 6 & $4 / 7$ & $100 \mathrm{u}$ & $97,689 \mathrm{u}$ & 2,310 & $60,446^{\circ}$ & $58,724^{\circ}$ \\
\hline 7 & $4 / 5$ & $100 \mathrm{u}$ & $101,121 \mathrm{u}$ & 1,121 & $51,340^{\circ}$ & $51,958^{\circ}$ \\
\hline 10 & $8 / 11$ & $8 / 11(100) \mathrm{u}$ & $72,192 \mathrm{u}$ & 0,534 & $36,027^{\circ}$ & $35,753^{\circ}$ \\
\hline $11^{*}$ & $16 / 25$ & $16 / 25(100) \mathrm{u}$ & $66,264 \mathrm{u}$ & 2,264 & $32,619^{\circ}$ & $33,807^{\circ}$ \\
\hline $44^{*}$ & $1 / 7$ & $1 / 7(100) \mathrm{u}$ & $18,274 \mathrm{u}$ & 3,988 & $32,520 / 4$ & $34,795^{\circ} / 4$ \\
\hline
\end{tabular}


Con el método $\mathrm{M}_{4}$ de construcción del endecágono y realizando la bisectriz de cada ángulo se obtiene el icosakaidígono configurado con 20 ángulos $\alpha$ y 2 ángulos $\beta(2 \pi=20 \alpha+2 \beta)$, cuyas ecuaciones analíticas son las siguientes:

$$
\alpha=\frac{1}{2} \arctan \frac{16}{25} \text { y } \beta=\pi-5 \arctan \frac{16}{25} .
$$

Además, las coordenadas de los 22 vértices son:

$$
\begin{gathered}
\mathrm{u}_{1,4}=(1,0) ; \mathrm{u}_{\mathrm{n}+1,4}=(\cos \mathrm{n} \alpha, \operatorname{sen} \mathrm{n} \alpha) \\
\operatorname{con} \mathrm{n}=1 \div 20 \mathrm{y}_{22,4}=(\cos (20 \alpha+\beta), \operatorname{sen}(20 \alpha+\beta)) .
\end{gathered}
$$

En la Tabla 2 se muestran directamente las medias de los parámetros de las correlaciones de comparación entre $\mathrm{M}_{4}$ y los $\mathrm{R}_{\mathrm{i}}, \mathrm{i}=1 \div 22$.

El icosakaidígono del método $\mathrm{M}_{5}$ está configurado con 21 ángulos $\alpha$ y 1 ángulo $\beta(2 \pi=21 \alpha+\beta)$, cuyas ecuaciones analíticas son las siguientes:

$$
\alpha=2 \arctan \frac{1}{7} \text { y } \beta=2 \pi-42 \arctan \frac{1}{7} \text {. }
$$

Además, las coordenadas de los 22 vértices son:

$$
u_{1,5}=(1,0) \mathrm{y} \mathrm{u}_{\mathrm{n}+1,5}=(\cos n \alpha, \text { sen } n \alpha) \operatorname{con} \mathrm{n}=1 \div 21 \text {. }
$$

Se observa que el proceso $\mathrm{M}_{4}$ está configurado con diez ángulos iguales $\alpha$ y un ángulo diferente $\beta$ al resto, y que el proceso $M_{5}$ está configurado con 42 ángulos iguales a y dos ángulos diferentes $\beta$. En la construcción de cualquier instrumento existe una imprecisión, con lo que es necesario ajustar dicho utensilio.

\subsection{Método $\mathrm{M}_{6}$}

La construcción mediante los instrumentos astronómicos se ha definido como $\mathrm{M}_{6}$. El método permite operar mediante el recto de este instrumento, de manera que mediante la división angular de la esfera del astrolabio $360^{\circ} / 22=16,36^{\circ}$, o del cuadrante $90^{\circ} /(5+1 / 2)=16,36^{\circ}$, se puede determinar la figura del icosakaidígono. Con la construcción de estos astrolabios se subdividía el perímetro de la circunferencia de su esfera en 72 marcas, una cada $5^{\circ}$, realizada mediante la bisectriz del ángulo de $30^{\circ}$ y luego trisecando, con un método clásico aproximado, el ángulo de $15^{\circ}$. El problema es determinar la precisión de la división y la apreciación de un grado en el astrolabio. Así se puede operar con una construcción donde se determina el ángulo de $16,36^{\circ}$ y con la aproximación de $\left(16^{\circ}+1 / 3^{\circ}\right)$, con ello podríamos operar con 20 ángulos iguales de $16,33^{\circ}$ y dos desiguales de $16,70^{\circ}$. Este método podría corregir el error mediante reiteración y ajuste.

El icosakaidígono del método $\mathrm{M}_{6}$ está configurado con 21 ángulos $\alpha_{i}$ y 1 ángulo $\beta\left(2 \pi=\sum_{i=1}^{21} \alpha_{i}+\beta\right)$, cuyas ecuaciones analíticas son las siguientes:

$$
\begin{gathered}
\alpha_{i}=\frac{17 \pi}{66}-6 \arctan \left(\frac{2 \sin \frac{\pi}{24}}{2 \cos \frac{\pi}{24}+1}\right)+\varepsilon_{i} \\
y \beta=126 \arctan \frac{2 \operatorname{sen} \frac{\pi}{24}}{2 \cos \frac{\pi}{24}+1}-\frac{75 \pi}{22}-21 \varepsilon_{i} .
\end{gathered}
$$

En estas ecuaciones hemos considerado que el error cometido por el escultor al asignar intuitivamente la diferencia angular de $\Delta=\frac{\pi}{132} \operatorname{rad}=1, \widehat{36}^{\circ}$ es $\varepsilon_{\mathrm{i}}=\frac{0,1 \pi}{180}$.
Por lo tanto las expresiones analíticas quedan de la siguiente manera:

$$
\begin{gathered}
\alpha=\frac{17 \pi}{66}-6 \arctan \frac{2 \operatorname{sen} \frac{\pi}{24}}{2 \cos \frac{\pi}{24}+1}+\frac{0,1 \pi}{180} \\
\text { y } \beta=126 \arctan \frac{2 \operatorname{sen} \frac{\pi}{24}}{2 \cos \frac{\pi}{24}+1}-\frac{75 \pi}{22}-\frac{21 \cdot 0,1 \pi}{180} .
\end{gathered}
$$

Además, las coordenadas de los 22 vértices son: $\mathrm{u}_{1,5}=(1,0)$ y $u_{n+1,5}=(\cos n \alpha$, sen $n \alpha) \operatorname{con} n=1 \div 21$.

\subsection{Método $M_{7}$}

El método de construcción directa $\mathrm{M}_{7}$ parte del triángulo equilátero $\mathrm{ABC}$, trazamos un segmento paralelo e igual al lado $\overline{\mathrm{AB}}$ que pase por el vértice $\mathrm{C}$, completando el rectángulo ABDE. Trazando las diagonales de este rectángulo encontramos $\mathrm{O}$, centro de la circunferencia que circunscribe el polígono. Prolongando la recta que pasa por $\mathrm{O}$ y $\mathrm{C}$, hasta que intercepte con la circunferencia, encontramos el punto F. Con centro D y radio $\overline{\mathrm{DF}}$ trazamos seis arcos consecutivos de circunferencia, encontrando así G, H, I, J, K, y L. De la misma manera y con el mismo radio, con centro en L, trazamos dos arcos determinando los puntos $\mathrm{M}$ y N. Con centro A y radio $\overline{\mathrm{DF}}$ encontramos sobre la circunferencia el punto $\mathrm{P}$. Con centro B y radio $\overline{\mathrm{DF}}$ trazamos ocho arcos consecutivos de circunferencia encontrando así los puntos restantes Q, R, S, T, U, V, W y X. Se observa que este proceso está configurado con catorce ángulos iguales $\alpha$ y ocho ángulos diferentes $\beta$ al resto (Figura 7).

El icosakaidígono del método $M_{7}$ está configurado con 14 ángulos a y 8 ángulos $\beta$, cuyas ecuaciones analíticas son las siguientes:

$$
\alpha=\pi-4 \arctan \frac{\sqrt{3}}{2} \text { y } \beta=-\frac{3 \pi}{2}+7 \arctan \frac{\sqrt{3}}{2} .
$$

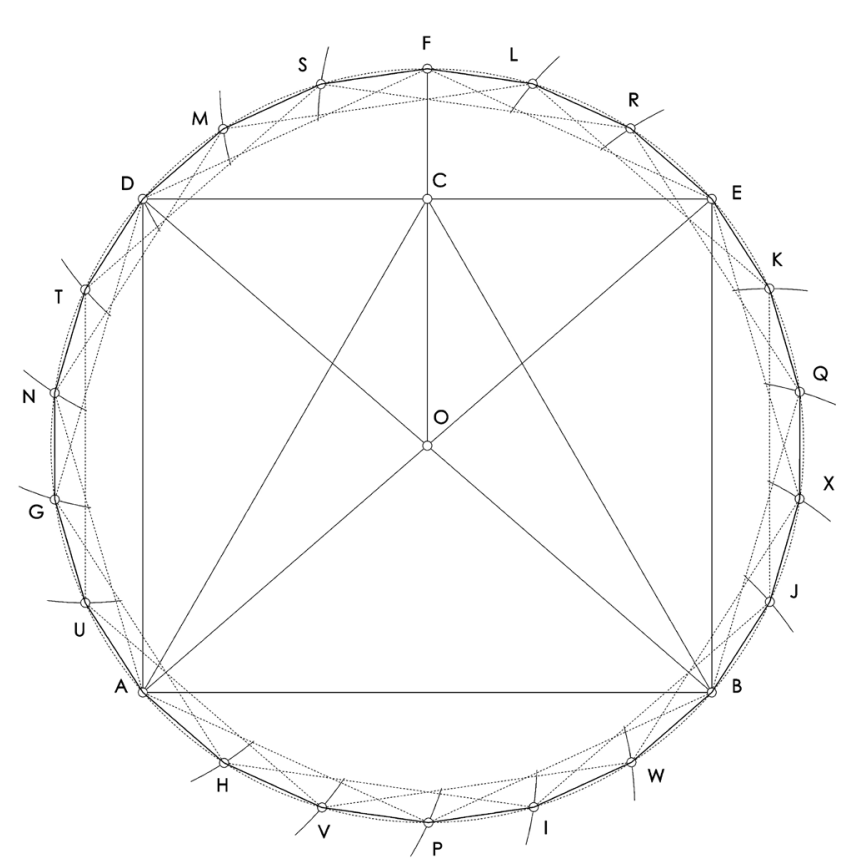

Figura 7. Construcción geométrica del método $M_{7}$. 
Además, las coordenadas de los 22 vértices son: $\mathrm{u}_{1,11}=(1,0)$; $\mathrm{u}_{\mathrm{n}+1,11}=(\cos \mathrm{n} \beta$, sen $\mathrm{n} \beta) \operatorname{con} \mathrm{n}=1 \div 2 ; \mathrm{u}_{\mathrm{n}+2,11}=(\cos (\beta+\mathrm{n} \alpha)$, $\operatorname{sen}(\beta+n \alpha)) \operatorname{con} n=2 \div 4 ; u_{n+3,11}=(\cos (2 \beta+n \alpha)$, sen $(2 \beta+n \alpha))$ $\operatorname{con} n=4 \div 5 ; u_{n+4,11}=(\cos (3 \beta+n \alpha)$, sen $(3 \beta+n \alpha)) \operatorname{con} n=5 \div 7$; $\mathrm{u}_{\mathrm{n}+5,11}=(\cos (4 \beta+\mathrm{n} \alpha)$, sen $(4 \beta+\mathrm{n} \alpha)) \operatorname{con} \mathrm{n}=7 \div 9 ; \mathrm{u}_{\mathrm{n}+6,11}=$ $=(\cos (5 \beta+n \alpha), \operatorname{sen}(5 \beta+n \alpha)) \operatorname{con} n=9 \div 11 ; u_{n+7,11}=(\cos (6 \beta+$ $+n \alpha)$, sen $(6 \beta+n \alpha))$ con $n=11 \div 12 y_{n+8,11}=(\cos (7 \beta+n \alpha)$, $\operatorname{sen}(7 \beta+n \alpha)$ ) con $n=12 \div 14$.

\section{DISCUSIÓN, ANÁLISIS DE LOS COEFICIENTES DE PEARSON}

Una vez analizados los siete métodos de análisis de las hipótesis $\left(\mathrm{M}_{1} \ldots \mathrm{M}_{7}\right)$, se procede a la comparación de éstos en función de las medias de los coeficientes Ajustados de Determinación de Pearson. El uso de dichos coeficientes tiene sentido siempre que sean altamente representativos; esta característica se mide con el Coeficiente de Variación de Pearson CV. De forma estándar, estadísticamente se considera que la media es representativa si CV $<25 \%$; haciendo los cálculos con todos los parámetros anteriores observamos que los CV obtenidos para las medias de los Coeficientes ajustados $\eta_{\text {adj }}^{2} \times 100$ son todas menores que 0,052\%. Esta altísima representatividad permite disponer con todos los resultados, geométricos y estadísticos, de las 154 comparaciones, por pieza. La más aproximada es la que se hace con el modelo $M_{7}$, en ambos casos según la Tabla 4 (Figura 8).

\section{CONCLUSIÓN}

El llamado icosakaidígono, polígono de 22 lados, no aparece en los Elementa de Euclides, ni en el Almagesto de Tolomeo, ni en las escasas Practica Geometriae de la época, pero puede ser construido mediante diferentes aproximaciones. Desde el punto de vista estadístico el método que más se aproxima en la construcción de las esculturas del Clypeus Tarragona, JupiterAmmon (s. I), y el del rosetón de la catedral Orvieto, con la escultura de la faz de Cristo (s. XIv), es el $\mathrm{M}_{7}$, utilizado probablemente en la construcción del Reliquiario del Corporale del Miracolo di Bolsena (1337-1338) de la catedral de Orvieto.

Por otra parte existe una extraordinaria coincidencia métrica entre ambas figuras, el Clypeus tiene una circunferencia de regresión de $\mathrm{R}=0,3349 \mathrm{~m}$, mientras que la de la faz de Cristo es de $\mathrm{R}=0,3378 \mathrm{~m}$. Los lados teóricos del icosakaidígono también se aproximan enormemente, en el primer caso 0,0953 m y en el rosetón o,0961 m. La composición del cuerpo central la figura de la faz de Cristo tiene la misma alegoría que los Clypeus respecto al dios Jupiter-Ammon, es como si esta conciencia compositiva hubiera perdurado desde la tradición clásica, a lo largo de más de un milenio, hasta el gótico.

En el resultado estadístico en segunda posición aparece la posible construcción mediante escuadras por rotación del $\mathrm{M}_{4}$, método que con su progresivo ajuste podría llegar a mejorar su precisión para el trazado de las esculturas.

Tabla 4. Ranking descendiente de comparación.

\begin{tabular}{|c|c|c|c|c|c|c|c|}
\hline \multicolumn{4}{|c|}{ Comparación con el rosetón } & \multicolumn{3}{c|}{ Comparación con el Cypleus } \\
\hline Métodos & $\mathbf{P}$ & $\eta_{\text {adj }}^{\mathbf{2}} \times \mathbf{1 0 0}$ & $\mathbf{d}$ & Métodos & $\mathbf{P}$ & $\eta_{\text {adj }}^{\mathbf{2}} \times \mathbf{1 0 0}$ & d \\
\hline $\mathrm{M}_{7}$ & 0,999938 & 99,986940 & 0,00034 & $\mathrm{M}_{7}$ & 0,990233 & 98,069530 & 0,00013 \\
\hline $\mathrm{M}_{4}$ & 0,999883 & 99,975434 & 0,00205 & $\mathrm{M}_{4}$ & 0,990187 & 97,968161 & 0,00203 \\
\hline $\mathrm{M}_{3}$ & 0,999853 & 99,969185 & 0,00215 & $\mathrm{M}_{3}$ & 0,990140 & 97,953991 & 0,00212 \\
\hline $\mathrm{M}_{6}$ & 0,999761 & 99,949825 & 0,00308 & $\mathrm{M}_{1}$ & 0,990047 & 97,941166 & 0,00391 \\
\hline $\mathrm{M}_{1}$ & 0,999760 & 99,949672 & 0,00396 & $\mathrm{M}_{5}$ & 0,990021 & 97,935968 & 0,00362 \\
\hline $\mathrm{M}_{5}$ & 0,999707 & 99,938510 & 0,00366 & $\mathrm{M}_{6}$ & 0,990045 & 97,933191 & 0,00305 \\
\hline $\mathrm{M}_{2}$ & 0,998850 & 99,758999 & 0,00864 & $\mathrm{M}_{2}$ & 0,989124 & 97,738024 & 0,00855 \\
\hline
\end{tabular}
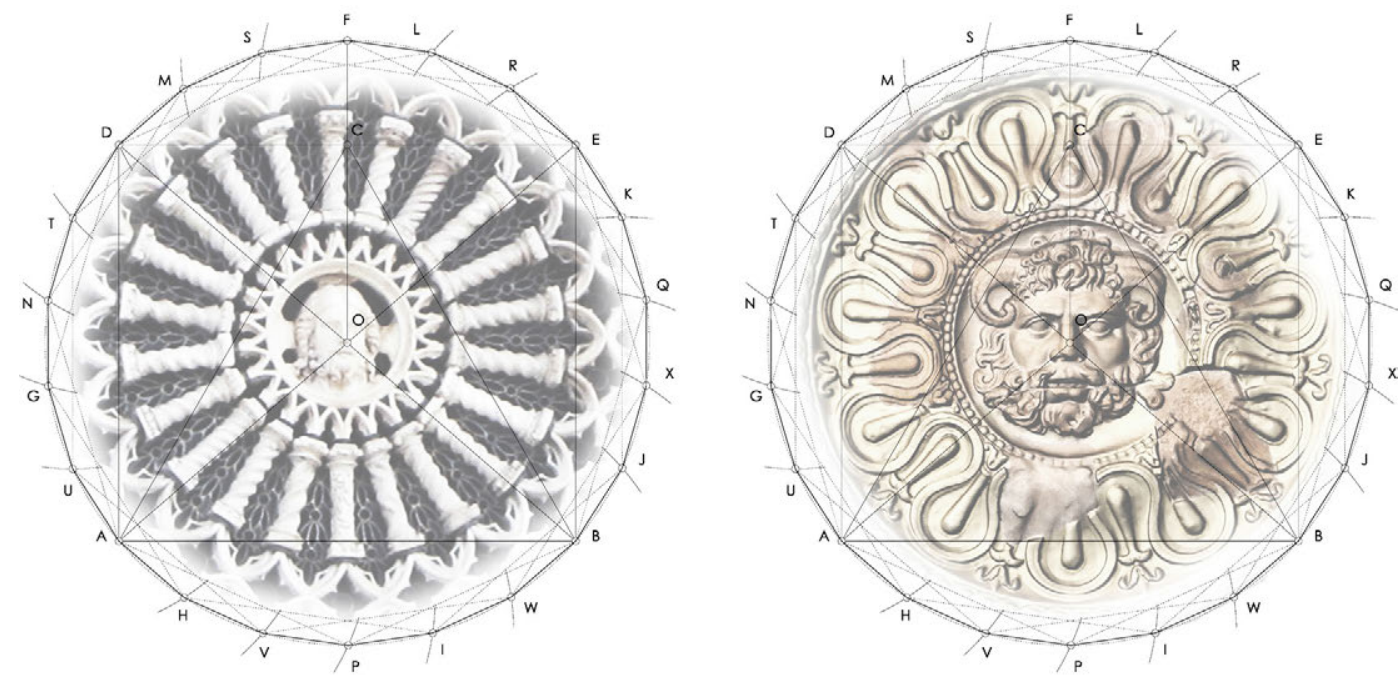

Figura 8. Construcción geométrica $\mathrm{M}_{7}$ del Corporale del Miracolo di Bolsena. 


\section{REFERENCIAS}

(1) Koppel, E. M. (1987). Relieves arquitectónicos en Tarragona. En Trillmich, W., Zanker, P. (coord.), Stadtbild und Ideologie die Monumentalisierung hispanischer Städte zwischen Republik und Kaiserzeit: Kolloquium in Madrid vom 19. bis 23. Oktober 1987. Bayerische Akademie der Wissenschaften. Munich: Supplements, New series 103, 1990, pp. 327-340.

(2) Peña Jurado, A. (2009). La decoración escultórica. En Ayerbe, R., Barrientos, T., Palma, F., El Foro de Augusta Emerita: génesis y evolución de sus recintos monumentales. Anejos de Archivo Español de Arqueología, LIII (pp. 583-622). Mérida: CSIC.

(3) Ascani, V. (1997). Il Trecento Disegnato. Le Basi Progettuali Dell'architettura Gotica in Italia, pp. 67-82. Roma: Viella.

(4) Bonelli, R. (1951). I disegni per la facciata del Duomo di Orvieto (1944). Bollettino dell'Istituto Storico-Artistico Orvietano, VII: $1-25$.

(5) Damiani, M. (1991). I restauri di Giuseppe Valadier alla facciata del duomo di Orvieto. Architettura, Storia E Documenti (pp. 260-274).

(6) Gillerman, D. M. (1994). The Evolution of the Design of Orvieto Cathedral, ca. 1290-1310. Journal of the Society of Architectural Historians, 53: 300-321, https://doi.org/10.2307/990939.

(7) Lassus, J. B. A. (1858). Album de Villard de Honnecourt. Architecte du XIIIe Siècle. Paris: Imprimerie impériale. PL XXIX y PL XXX.

(8) Ascani, V. (1989). Le dessin d'architecture médiéval en Italie. En Les Batîsseurs de Cathédrales Gothiques (pp. 255-277). Strasbourg: Musées de la ville de Strasbourg.

(9) Bork, R. (2005). Plan B and the Geometry of Façade Design at Strasbourg Cathedral, 1250-1350. Journal of the Society of Architectural Historians, 64: 442-473, https://doi.org/10.2307/25068200.

(10) Recht, R. (1989). Les Batîsseurs de Cathédrales Gothiques, pp. 393-397. Strasbourg: Musées de la ville de Strasbourg.

(11) Bucher, F. (1979). Architector. The Lodge Books and Sketchbooks of Medieval architects, vol. I, pp. 209, 216, $222,268$. New York: Abaris Books.

(12) Heath, T. L. (1908). The Thirteen Books of Euclid's Elements, vol. II, pp. 91-95. Cambrige: Cambridge University Press.

(13) Toomer, G. J. (1984). Ptolemy's Almagest, pp. 35-74. Translated and annotated by G. J. Toomer. With a foreword by Owen Gingerich. London: Gerald Duckworth \& Co.

(14) Branner, R. (1960). Villard de Honnecourt, Archimedes, and Chartres. Journal of the Society of Architectural Historians, XIX: 91-96, https://doi.org/10.2307/988023.

(15) Heath, T. L. (1921). A History of Greek Mathematics, Vol. II: From Aristarchus to Diophantus, pp. 328-329. Oxford: The Clarendon Press.

(16) Schoene, H. (1903). Heronis Alexandrini Opera Quae Supersunt Omnia. Vol III: Rationes Dimetiendi et Commentatio Dióptrica, p. 63. Leipzig: Teubner.

(17) Toldrà, J. M., et al. (2014, 12-14 de junio). The octagon, the hendecagon and the approximation of pi: the geometric design of the clypeus in the enclosure of Imperial cult in Tarraco. En XII International Forum, Le vie dei Mercanti, Best practice in heritage conservation management, from the world to Pompeii. Aversa, Capri.

(18) Wilson Jones, M. (2000). Principles of Roman Architecture, p. 75. 3. ${ }^{\text {a }}$ edición. New Haven and London: Yale University Press.

(19) Bechmann, R. (1991). Villard de Honnecourt. La pensée tecnique au XIIIe siècle et sa communication, pp. $146-148$. Paris: Picard Éditeur.

(20) Sarrade, M. T. (1986). Sur les connaissances mathématiques des bâtisseurs de cathédrales, pp. 48-54. Paris: Librairie du Compagnonnage.

(21) Nuere, E. (2001). Nuevo tratado de la carpintería de lo blanco, p. 68. Madrid: Ediciones Munilla-Leira.

(22) Pez, B. (1853). Hermanni Contracti monachi Augiensis de mensura astrolabii liber. Patrologia Latina, vol. 143, MPL143, Col. 0379-0390A.

(23) Baron, R. (1956). Hugonis de Sancto Victore Practica geometriae. Osiris, 12: 176-224, https://doi.org/10.1086/368598.

(24) Victor, S. K. (1979). Practical geometry in the high middle ages. Artis cuiuslibet consummatio and the pratike de geometrie, pp. 107-471. Philadelphia: The American Philosophical Society.

(25) Hahn, N. L. (1982). Medieval Mensuration: "Quadrans vetus" and "Geometrie due sunt partes principals", pp. 6-113. Transactions of the American Philosophical Society. Philadelphia: The American Philosophical Society.

(26) Busard, H. L. L. (1965). The Practica Geometriae of Dominicus de Clavasio. Archive for History of Exact Sciences, 2: 520-575, https://doi.org/10.1007/BFoo324882.

(27) Beaujouan, G. (1975). Réflexions sur les rapports entre théorie et pratique au Moyen Âge. En Murdoch, J. E., Sylla, E. D. (Eds.), The Cultural Context of Medieval Learning (pp. 437-484). Dordrecht, Boston: D. Reidel, https://doi. org/10.1007/978-94-010-1781-7_12.

(28) Barbella, G. (2010). Incendio di Borgo di Raffaello. Il rosone del misterio Trinitario. Brescia.

(29) Armesto, J., et al. (2010). Modelling masonry arches shape using terrestrial laser scanning data and nonparametric methods. Engineering Structures, 32: 607-615, https://doi.org/10.1016/j.engstruct.2009.11.007.

(30) Núñez, A., et al. (2012). Generation of virtual models of cultural heritage. Journal of Cultural Heritage, 13: 103-106, https://doi.org/10.1016/j.culher.2011.06.004.

(31) Alkan, R. M., et al. (2012, 6-10 May). Analysis of The Accuracy of Terrestrial Laser Scanning Measurements. En FIG Working Week 2012. Rome, Italy.

(32) Serrano, F., Saumell, J., Berenguer, F. (2014). Análisis de resultados métricos de una nube de puntos y una medición directa en el patrimonio edificado. El Santuario de La Montaña en Cáceres. Informes de la Construcción, 66(534): eo16, doi: http://dx.doi.org/10.3989/ic.12.059. 
(33) Pavlidis, G., Koutsoudis, A., Fotis, A., Vassilios, T., \& Christodoulos, C. (2007). Methods for 3D digitization of Cultural Heritage. Journal of Cultural Heritage, 8(1): 93-98, http://doi.org/10.1016/j.culher.2006.10.007.

(34) Yilmaz, H. M., Yakar, M., Gulec, S. A., \& Dulgerler, O. N. (2007). Importance of digital close-range photogrammetry in documentation of cultural heritage. Journal of Cultural Heritage, 8(4): 428-433, https://doi.org/10.1016/j.culher.2007.07.004.

(35) McCarthy, J. (2014). Multi-image photogrammetry as a practical tool for cultural heritage survey and community engagement. Journal of Archaeological Science, 43: 175-185, https://doi.org/10.1016/j.jas.2014.01.010.

(36) Koutsoudis, A., Vidmar, B., Ioannakis, G., Arnaoutoglou, F., Pavlidis, G., \& Chamzas, C. (2014). Multi-image 3D reconstruction data evaluation. Journal of Cultural Heritage, 15(1): 73-79, https://doi.org/10.1016/j.culher.2012.12.003.

(37) Souto-Vidal, M., Ortiz-Sanz, J., Gil-Docampo, M. (2015). Implementación del levantamiento eficiente de fachadas mediante fotogrametría digital automatizada y el uso de software gratuito. Informes de la Construcción, 67(539): e107, doi: http://dx.doi.org/10.3989/ic.14.098.

(38) Chandler, J. H., Fryer, J. (2013). Autodesk 123D Catch: How accurate is it? Low cost digital photogrammetry. Geomatics World, January/February, http://geomaticsjc.lboro.ac.uk/ven/webpages/OtherFiles/Chandler,\%20Fryer\%20-\%20 2013\%20-\%20AutoDesk\%20123D\%20Catch\%20How\%20accurate\%20is\%20it.pdf

(39) McCarthy, J. (2014). Multi-image photogrammetry as a practical tool for cultural heritage survey and community engagement. Journal of Archaeological Science, 43(1): 175-185, http://doi.org/10.1016/j.jas.2014.01.010. 